\title{
IONIZATION PHENOMENA IN GASES
}

$\mathrm{T}$ HE Physical Society, the Institute of Physics and the Electrical Research Association were the sponsors of the first international conference on "Ionization Phenomena in Gases", held at the Clarendon Laboratory, Oxford, during July 18-23. The 250 visitors included many distinguished foreign guests from ten countries. Social activities were held over the week-end, beginning with a reception at Christ Church at which Lord Cherwell and Sir John Townsend were present. There were conducted tours of Oxford colleges and a garden party given by the British Council for overseas visitors. Later in the week ladies visited Blenheim Palace, a blanket mil at Witney and the theatre at Stratford-upon-Avon. Receptions were also given by the British Electricity Authority, and by the Mayor of Oxford.

The conference was opened by Dr. A. von Engel, who surveyed the work in Great Britain. The delegates were also welcomed by Mr. T. C. Keeley, director of the Clarendon Laboratory. Chairmen at the sessions were, respectively: Dr. S. Whitehead, Dr. C. Sykes, Mr. R. Davis, Mr. E. W. B. Gill, Dr. E. J. Bowen, Sir John Cockeroft, Dr. T. E. Allibone and Prof. R. Whiddington.

The main subjects discussed were spark, arc, glow and high-frequency discharges, Geiger counters and several fundamental processes.

There were two schools of thought on the formation of sparks : one ascribing their development to photoelectric action in the gas aided by multiplication in the electric field; the other to secondary processes at the cathode again aided by Townsend multiplication. In support of the first, J. D. Craggs (Liverpool) gave measurements of the rate of the radial expansion of spark channels at high pressure. $\mathrm{He}$ discussed how closely thermal equilibrium is attain $\theta$ d in the spark channel and noted the observed differences in the 'excitation temperatures' of the various emission lines. J. M. Meek and R. F. Saxe (Liverpool) showed from their measurements on sparks up to $100 \mathrm{~cm}$. long how streamers grow in time and space just before a final breakdown.

The other idea is that Townsend's theory adequately describes all the conditions leading to breakdown, and applies equally to low and high pressures. F. Llewellyn Jones (Swansea) reviewed this theory and showed that, with extremely stable cathode conditions and electric fields, ionization currents increase with distance as predicted by Townsend. This is true even when pressure is greater than $760 \mathrm{~mm}$. mercury and up to voltages approaching the breakdown value. In the discussion, he suggested that in impulse fields, luminosity, which depends on high ion density, may appear at the anode, later moving quickly towards the cathode. C. Grey Morgan (Swansea) stressed the importance of field emission caused by surface layers on metal cathodes.

L. H. Fisher (New York) described the marked effect of excess voltage on the formative time lag. When the voltage is just above threshold, the lag is about $10^{-4}$ sec. in air, nitrogen, argon and oxygen. This again supports Townsend's theory and points to photoelectric emission at the cathode, except in argon, where there is a long delay even at high excess voltages, probably due to imprisonment of resonance radiation. C. E. R. Bruce (Electrical Research Association) pointed out that breakdown fields in air for extremely long gaps (for example, between clouds and earth) are about $100 \mathrm{~V} . / \mathrm{cm}$., which shows that the starting process must be different from that with uniform fields and metal electrodes. Corona currents which flow radially outwards from streamers were mentioned, and certain solar eruptions were likened to long sparks.

In the session on arc discharges, R. Mannkopff (Göttingen) discussed radiation equilibria. By examining spectral intensities he showed that after an arc is switched off, thermal excitation and ionization persist, and must have predominated previously. Auto-ionization appears to be a probable process. W. Lochte-Holtgreven (Kiel) described high-power ares in water vapour giving temperatures of about $50,000^{\circ} \mathrm{K}$. The electron concentration is determined by the equation of Inglis and Teller, after observing the number of Balmer lines of hydrogen visible. The continuous background is partly caused by negative atomic hydrogen ions. $H$. Maecker (Erlangen) showed how, by varying the pressure in such arcs between 100 and 1,000 atmospheres, one can discriminate between, and measure, the cross-sections for electrons colliding with neutral atoms and positive ions. M. Hoyaux (Charleroi) described the use of Ledrus's 'plasmograph', recording Langmuir's probe characteristics in mercury rectifier discharges with a time resolution of less than 20 microsec.

P. F. Little (Oxford) opened the session on glow discharges by describing how the electric field distribution in a hollow cathode discharge in nitrogen can be measured. The deflexions of an electron beam show a linear fall of field with distance from the cathode down to very small values at the boundary of the glow. 'The large current density observed is explained by a large ion concentration and ion-drift velocity. He concluded that many electrons are emitted from the cathode by light from the intense glow. W. Fucks (Aachen) has used corona discharges to measure aerodynamic effects, notably the appearance of vortices, and the associated flow of gas. W. L. Harries (Oxford) discussed the abnormally low starting fields in high-frequency electrodeless discharges in neon; he attributes them to the production of a large concentration of excited atoms which are later ionized by low-energy electrons released by light from the glass walls. G. Valle (Bologna) has applied relaxation oscillations of increasing frequency to a discharge in argon or neon. The restriking potentials decrease when the residual ionization from the afterglow becomes important. W. A. Prowse (Durham) showed that in microwave discharges ionization is due to electron collisions and loss to diffusion. With short pulses, however, a critical electron density has to be reached within the pulsetime to obtain breakdown. In polyatomic gases, a formative lag of less than $10^{-8}$ sec. is observed. It is suggested that the oscillating electrons produce excited molecules which are readily ionized. This idea is being tested by applying an auxiliary D.c. electric field at right angles which drives the electrons into unexcited gas. Oscillograms of the current in pulsed high-frequency electrodeless discharges were presented by G. Francis (Oxford), who discussed the mechanism of growth of current. He showed that in hydrogen two secondary processes, one fast and one 
slow, are nəcessary. S. C. Brown (Massachusetts Institute of Technology), injecting electrons into a low-pressure mercury plasma, observed plasma oscillations only when ion sheaths were present around the electrodes. Their frequency depended on the density of the electrons which oscillate in certain standing-wave forms. From the variation of the frequency with the thickness of the sheath, it follows that a 'transit-time process' causes these oscillations. D. C. Dalton (London) reported experiments which indicate the effect of space charges on the breakdown of high-frequency discharges in air.

In the following session, G. L. Weissler (Los Angeles) described his vacuum spectrographic measurements in rare and molecular gases, which yield the absorption coefficients as a function of the wave-length and thus the photo-ionization crosssections. Preliminary results have also been quoted of photoelectric vields on various metal surfaces, again for wave-lengths between 100 and $1000 \mathrm{~A}$. In the discussion, W. B. Nottingham (Massachusetts Institute of Technology) mentioned some results obtained with pure silica. P. J. Jutsum (Reading) compared his experimental results of photo-ionization cross-sections in sodium, magnesium, calcium and thorium vapours with the calculated values and described a method of discriminating between molecular and atomic absorption. In a written communication, A. and E. Vassy (Paris) described the character of spectra of sparks in air of $15-150 \mathrm{~cm}$. length ; a study of the spectral lines as a function of the time has also been made. G. Valle (Bologna), in a paper read by R. W. Lunt, described his theory of breakdown in a transverse magnetic field. This field is supposed to have the same effect as an increase in density of gas. Assuming only inelastic collisions with electrons of constant mean free path, he obtains results which agree roughly with experiments. J. M. Somerville (Sydney), extending this theory, included the distribution of free paths and the partial capture of electrons driven back to the cathode by the field. $\mathrm{He}$ also described the effect of transverse fields on the length of the dark space in glow discharges between concentric cylinders.

In the session on counters, R. Loosemore (Harwell), in a paper read by W. Abson, discussed certain features of the bromine-quenched counter. At a critical voltage a second pulse appears, due to another discharge between ion sheath and cathode. The probable origin of spurious oscillations was also discussed. P. J. Campion (Oxford) showed cloudchamber photographs of the propagation of a Geiger discharge along a counter wire in an air-alcohol mixture; the charge distribution within the ion sheath was measured. H. den Hartog (Amsterdam) spoke about the electron component in the Geiger discharge, which he thinks may be explained by the large radius of the space charge at high excess voltages. A group of Italian workers, L. Colli, U. Facchini, G. Bertolini and others (Milan), presented papers on the mechanism of argon-filled counters. $\alpha$-Particles were used to trigger light and electron current pulses which were observed simultaneously. The light pulse lasts longer, due to the long life of metastable argon atoms and excited molecules. Current oscillograms from counters with nickel or brass eathodes show that their photoelectric yield must be of the order of $10^{-2}$. Ionization currents in argon with small amounts of polyatomic gases were found to differ from those theoretically expected. This is sometimes due to interaction between the components of the mixture, sometimes to ionization by $\delta$-rays (slow electrons).

High-vacuum studies were introduced by R. L. Fortescue (London), who talked about breakdown in highly evacuated gaps. Bursts of current precede the development of an arc and depend on the condition of both electrodes. J. W. Leech (London) presented calculations on these pulses, assuming that the electrons are confined to narrow channels, and estimated the gas pressure necessary to make them diverge. D. Alpert (Pittsburgh) reviewed the existing ultra high-vacuum technology and described new methods of obtaining a pressure so low as $10^{-10} \mathrm{~mm}$. mercury without getters or refrigerants. S. Wagener (General Post Office, London) showed that the high gettering rates of barium can only be understood by assuming that the reacting gas particles are atoms, not molecules. Selective getters were also treated. J. H. Mason (Electrical Research Association) showed oscillograms and Lichtenberg figures of pulsedischarges in cavities in dielectrics when subjected to low-frequency fields and offered tentative explanations.

In the last session, R. Geballe (Seattle) described simultaneous measurements of ionization and attachment coefficients and discussed quantitatively the effect on the ionization current as a function of the electrode separation. N. L. Oleson (Monterey) presented preliminary work using Langmuir probes and photomultipliers for measuring sinultaneously charge density and light from striations in an argon positive column. Kantrowitz (Cornell) reported measurements on ionization and light produced in argon and in argon-oxygen mixtures by shock waves.

$$
\text { A. VON ENGEL }
$$

G. Francis

\section{GROUP DISCUSSION}

$\mathrm{N}$ view of the increasing use which is being made of discussion groups as an educational medium, the editors of the Health Education Journal are to be commended for devoting the whole of a rec?nt issue to the subject of group discussion $(11$, No. 3 ; July 1953). The theory of group discussion is introduced by Dr. M. L. Johnson, who suggests that the disadvantage of the lecture as a teaching method is that there is no 'feed-back' to the teacher; he cannot know what is selected or how what is absorbed is distorted. $\mathrm{He}$ can only try to present the material in a manner which, from his judgment of the listeners' backgrounds, he considers interesting and unambiguous, so that they will absorb as much, and in as undistorted a form, as possible. This disadvantage does not apply to the discussion method; but, to be successful, discussion must be carried out in a "permissive atmosphere". This is essential because people must not be allowed to feel afraid of making fools of themselves by exposing the irrationality of their thinking and feeling. They must feel free to talk in a spontaneous, even incoherent and random manner, so that assumptions can be brought to light and their usefulness examined.

The means of introducing discussion is examined by Dr. Emrys Davies, who indicates that, in most cases, discussion is educational in character because almost invariably it aims at modifying attitudes and changing behaviour. To be fully effective the leader of the discussion group should devote considerable time to preparation before meeting his group. Besides 